Article

\title{
Research on Fabrication Techniques and Focusing Characteristics of Metalens
}

\author{
Yuhui Zhang $1,2,3, *\left(\mathbb{0}\right.$, Yuegang Fu ${ }^{1,2,3,+}\left(\mathbb{0}\right.$, Chenhao Ma ${ }^{1,2,3,+}$, Bowei Yang ${ }^{1,+}$ and Yuanzhi Zhao ${ }^{1,+}$ \\ 1 School of Optoelectric Engineering, Changchun University of Science and Technology, \\ Changchun 130022, China; fuyg@cust.edu.cn (Y.F.); mach@cust.edu.cn (C.M.); \\ 2020200040@mail.cust.edu.cn (B.Y.); zyzzyzzyz0331@163.com (Y.Z.) \\ 2 Key Laboratory of Optoelectronic Measurement and Optical Information Transmission Technology of \\ Ministry of Education, Changchun University of Science and Technology, Changchun 130022, China \\ 3 Key Laboratory of Advanced Optical System Design and Manufacturing Technology of the Universities of \\ Jilin Province, Changchun 130022, China \\ * Correspondence: 2022800005@cust.edu.cn \\ + These authors contributed equally to this work.
}

check for updates

Citation: Zhang, Y.; Fu, Y.; Ma, C.; Yang, B.; Zhao, Y. Research on Fabrication Techniques and Focusing Characteristics of Metalens. Coatings 2022, 12, 359. https://doi.org/ 10.3390/coatings12030359

Academic Editors: Anna Palau and Anatoly Kovalev

Received: 25 January 2022

Accepted: 3 March 2022

Published: 8 March 2022

Publisher's Note: MDPI stays neutral with regard to jurisdictional claims in published maps and institutional affiliations.

Copyright: (C) 2022 by the authors. Licensee MDPI, Basel, Switzerland. This article is an open access article distributed under the terms and conditions of the Creative Commons Attribution (CC BY) license (https:// creativecommons.org/licenses/by/ $4.0 /)$.

\begin{abstract}
Metalenses have recently attracted increased attention due to their remarkable characteristics. The fabrication technology of metalenses has also become an important research direction. In this study, we propose a metalens structure based on $\mathrm{Au}-\mathrm{MgF}_{2}-\mathrm{Au}$ in infrared waveband. The preparation process of the metalens included magnetron sputtering, electron beam evaporation, and electron beam exposure. A dose test was performed during the exposure process, adjusting the exposure dose to minimize the proximity effect after exposure. Then, SEM was used to measure the processed metalens structure, and FDTD software was used to build a model based on the metalens, simulating and analyzing its focusing characteristics. The results show that the size deviation produced during the processing has little effect on the functionality of the metalens. The processed metalens can also focus different polarized light incidences at different spatial positions: The metalens can focus at $4.97 \mu \mathrm{m}$ for x-polarized light and focus at $13.5 \mu \mathrm{m}$ for $\mathrm{y}$-polarized light. Additionally, the metalens has good focusing effects with different working wavelengths. We believe that the processing method of metalens proposed in this paper provides guidance for the preparation of subwavelength metasurface structures, and our findings are beneficial in developing new methods of near-infrared regime manipulation.
\end{abstract}

Keywords: metalens; preparation; infrared waveband; different polarization; focus

\section{Introduction}

Metasurfaces have characteristics that are not available in natural materials [1-9] and have obvious advantages in size, volume, processing $[10,11]$, etc., meeting the requirements of miniaturization and integration in modern technology, making them key research objects for a large number of scientific researchers. Metalens is a two-dimensional plane lens based on metasurfaces, which realizes the focusing function of a traditional lens by integrating the transmission phase or reflection phase [12-14]. Metalens is different from the traditional lens; it has a subwavelength size, which is advantageous to the processing and miniaturization of the device. Based on the different wavelength and polarization states of the incident light, metalens can achieve a variety of different functions [15-24]. It has important applications in telescopes, cameras, and other imaging fields, as well as tunable devices, holographic displays, terahertz imaging, and nonlinear optics, and has great potential for development in the micro-nano field. Although many metalens structures have been proposed before, their processing is more difficult and has rarely been elaborated in previous studies; therefore, the fabrication technology of metalens and focusing characteristics of the processed metalens have not been fully investigated. 
In this paper, we propose a metalens structure based on $\mathrm{Au}-\mathrm{MgF}_{2}-\mathrm{Au}$ in infrared waveband. The preparation process of the metalens includes magnetron sputtering (type, producer, city (state), country), electron beam evaporation, and electron beam exposure. A dose test was performed during the exposure process, adjusting the exposure dose to minimize the proximity effect after exposure. Then, a scanning electron microscope (SEM) was used to measure the processed metalens structure, and the finite-difference time-domain (Lumerical FDTD solutions 8.15.736.0) method was used to build a model based on the metalens, simulating and analyzing its focusing characteristics. The results showed that the size deviation produced during the processing has little impact on the functionality of the metalens. The processed metalens can focus on different polarized incidences at different spatial positions. For x-polarized incidence, the focal length is $4.97 \mu \mathrm{m}$. For $\mathrm{y}$-polarized incidence, the focal length is $13.5 \mu \mathrm{m}$. For 45 -degree linearly polarized incidence, there are two focal points, and the focal length are $4.97 \mu \mathrm{m}$ and $13.5 \mu \mathrm{m}$, respectively. For circularly polarized incidence, there are also two focal points, and the focal point intensity is twice that of 45-degree linearly polarized light. The processed metalens also has good focusing effects with different working wavelengths. We believe that the processing method of metalens proposed in this paper provides guidance for the preparation of subwavelength metasurface structures, and our findings are beneficial in developing new methods of near-infrared regime manipulation.

\section{Structural Design}

Figure 1 illustrates the schematic of the proposed metalens, which is a metal-insulatormetal structure to form a Fabry-Perot cavity to enhance the interaction between light and resonance antenna. The material for the resonance antenna and the bottom mirror was selected as $\mathrm{Au}$ [21], while the material for the dielectric spacer was chosen as $\mathrm{MgF}_{2}$ [22]. The thicknesses of $\mathrm{Au}$ antenna, $\mathrm{MgF}_{2}$ spacer, and $\mathrm{Au}$ mirror were set as $30 \mathrm{~nm}, 50 \mathrm{~nm}$, and $130 \mathrm{~nm}$, respectively. The length and width of the unit cell of the metalens were both $200 \mathrm{~nm}$. The x-direction of the metalens was composed of 60 unit structures, and the y-direction was periodically arranged for 30 cycles. The length and width of the resonance antenna can be calculated according to [14].

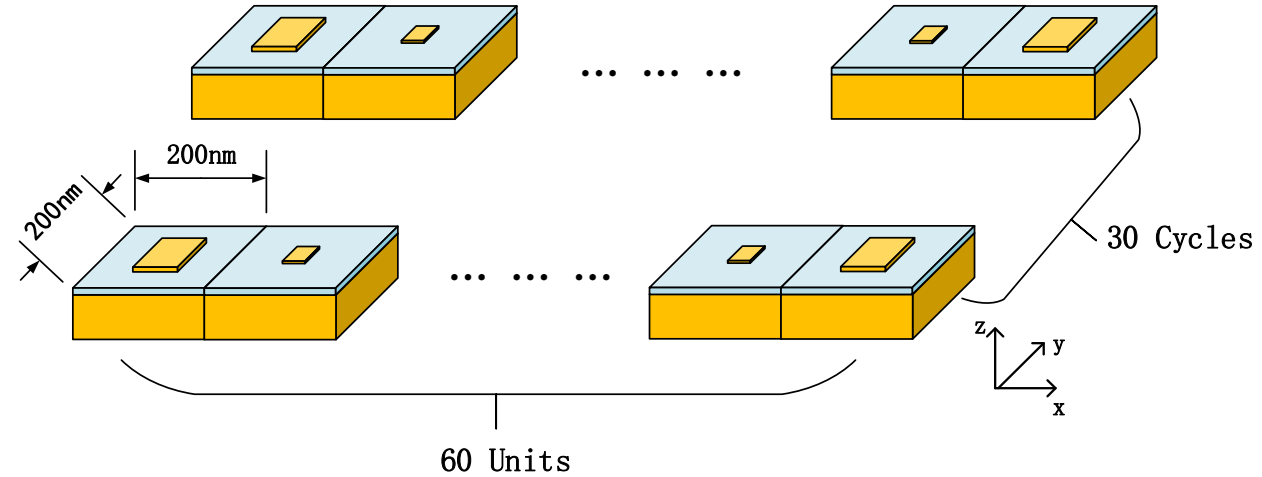

Figure 1. Diagram of the metalens structure.

The proposed structure can be designed to achieve the function of the focusing lens. To achieve desired focusing effect, the relative phase of the reflected wave at position $x$ from the center should follow the expression [12].

$$
\varphi(x)=\frac{2 \pi}{\lambda_{0}}\left(\sqrt{(x+\Delta x)^{2}+F^{2}}-F\right)
$$

where $\lambda_{0}$ is the incident wavelength, $F$ represents the designed focal length, and $\Delta x$ represents the shift of focal point along the $\mathrm{x}$-axis. 


\section{Preparation of the Structure}

The preparation process of the metalens is shown in Figure 2. First, the silicon wafer was ultrasonically cleaned with acetone-isopropanol-water and dried with nitrogen, and then, a $130 \mathrm{~nm}$ Au film was grown on the silicon wafer by magnetron sputtering (Lab18, Kurt J. Lesker, PA, USA). In the second step, a $\mathrm{MgF}_{2}$ film was deposited on the $\mathrm{Au}$ film by electron beam evaporation technology (Ei-5z, Ulvac, Kanagawa, Japan). The deposition rate and thickness of the $\mathrm{MgF}_{2}$ film material were monitored by a quartz crystal; the film deposition rate was controlled at $0.2 \mathrm{~nm} / \mathrm{s}$, and the deposition thickness was $50 \mathrm{~nm}$. In the third step, electron beam lithography (JBX5500ZA, JEOL, Tokyo, Japan) was used to prepare subwavelength Au nanoantennas. Here, the electron beam lithography technology mainly included the following key processes: spin coating, exposure, development, electron beam evaporation, stripping, etc. Spin coating photoresist on the surface of $\mathrm{MgF}_{2}$ film was divided into two steps. The first step was to apply MMA, and the second step was to apply PMMA. A layer of $\mathrm{Cr}$ film was grown by magnetron sputtering before exposure. The reason for growing the $10 \mathrm{~nm} \mathrm{Cr}$ film is to increase conductivity and reduce the proximity effect effectively. Then, the exposure and development processes began, during which the exposure dose varied from 100 to $1000 \mu \mathrm{C} / \mathrm{cm}^{2}$. Before developing, it was necessary to use a Cr etching solution to remove $\mathrm{Cr}$. The developer used a mixture of 4-Methyl-2-pentanone and isopropanol. After the above process was completed, the electron beam evaporation process was used to deposit $\mathrm{Au}$. To improve adhesion between $\mathrm{MgF}_{2}$ and $\mathrm{Au}, 5 \mathrm{~nm}$ of Ti was deposited as a binder, after which $25 \mathrm{~nm}$ Au was deposited. Finally, a peeling process was performed to obtain a metalens structure of $\mathrm{Au}-\mathrm{MgF}_{2}-\mathrm{Au}$.
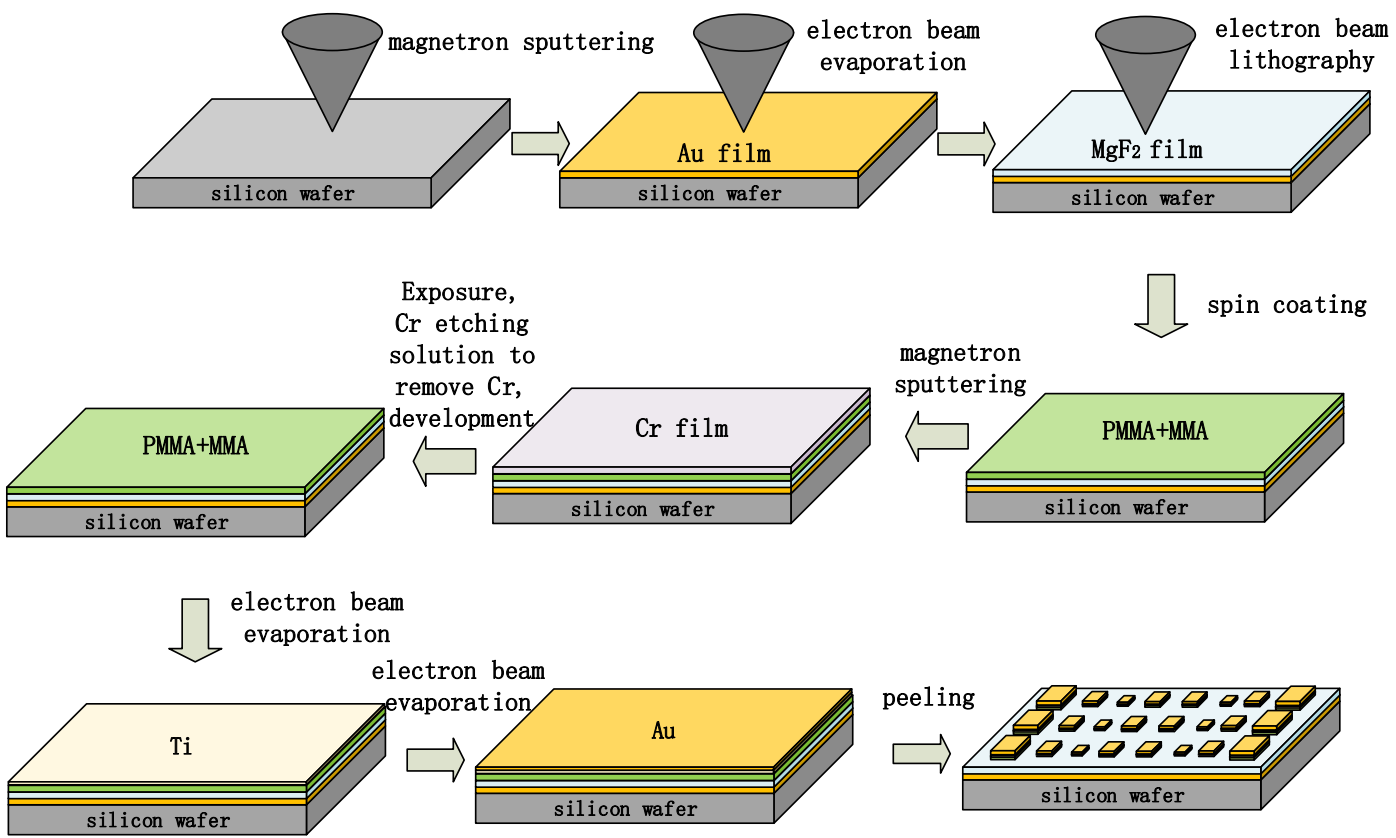

Figure 2. Process flowchart of the proposed metalens structure.

According to the above process, a batch of samples was processed, and the morphology was tested by the SEM (Eline Plus, Raith, Germany). The test results are shown in Figure 3. The results show that the effect of large size graphics is good, but the proximity effect of small size graphics is more significant. In electron beam lithography, due to the electron beam scattering in the glue layer and the substrate, outside the area where the glue layer is exposed, the adjacent areas are also exposed, resulting in the edge of the glue layer being blurred, and therefore, the shape of the graph expands to the adjacent areas, that is, the adjacent areas affected by the electron beam exposure. Therefore, it is necessary to dose and correct for the proximity effect before preparing samples. There are three different methods to correct the proximity effect of electron beam lithography: exposure dose correction, 
figure size compensation, and background exposure compensation. Among them, dose correction is the most widely used method, and the most effective method. Therefore, we conducted a dose test during the exposure process; in other words, we adjusted the exposure dose to minimize the proximity effect after exposure.
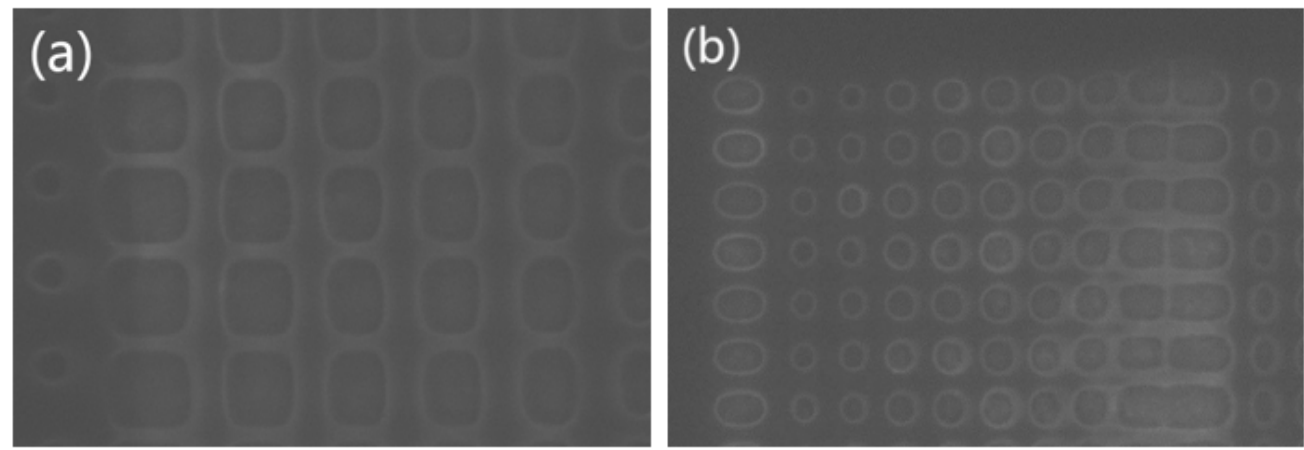

Figure 3. The scanning electron microscopic image (SEM) of samples: (a) the SEM image at $82,490 \times$ magnification; (b) the SEM image at $46,400 \times$ magnification.

Figure 4 illustrates the SEM testing of different exposure doses. Figure $4 \mathrm{a}$ is the SEM image with an exposure dose of $700 \mu \mathrm{C} / \mathrm{cm}^{2}$; the middle part of the image is convex and irregular, indicating that the exposure dose is too large. When the exposure dose is $300 \mu \mathrm{C} / \mathrm{cm}^{2}$, almost no image can be clearly presented, indicating that the dose is too small. Figure $4 \mathrm{~b}$ is the SEM image with an exposure dose of $400 \mu \mathrm{C} / \mathrm{cm}^{2}$; the proximity effect in the image is very small, but the morphology of small size graphics is not good. Figure $4 \mathrm{c}$ is the SEM image with an exposure dose of $500 \mu \mathrm{C} / \mathrm{cm}^{2}$; the impact of proximity effect on patterns in the image is seen to be very small, and the morphology of small size graphics is relatively good. Figure $4 \mathrm{~d}$ is the SEM image with an exposure dose of $600 \mu \mathrm{C} / \mathrm{cm}^{2}$; the graphics appear deformed, indicating that the exposure dose is now too large. Therefore, an exposure dose of $500 \mu \mathrm{C} / \mathrm{cm}^{2}$ was chosen to be used.

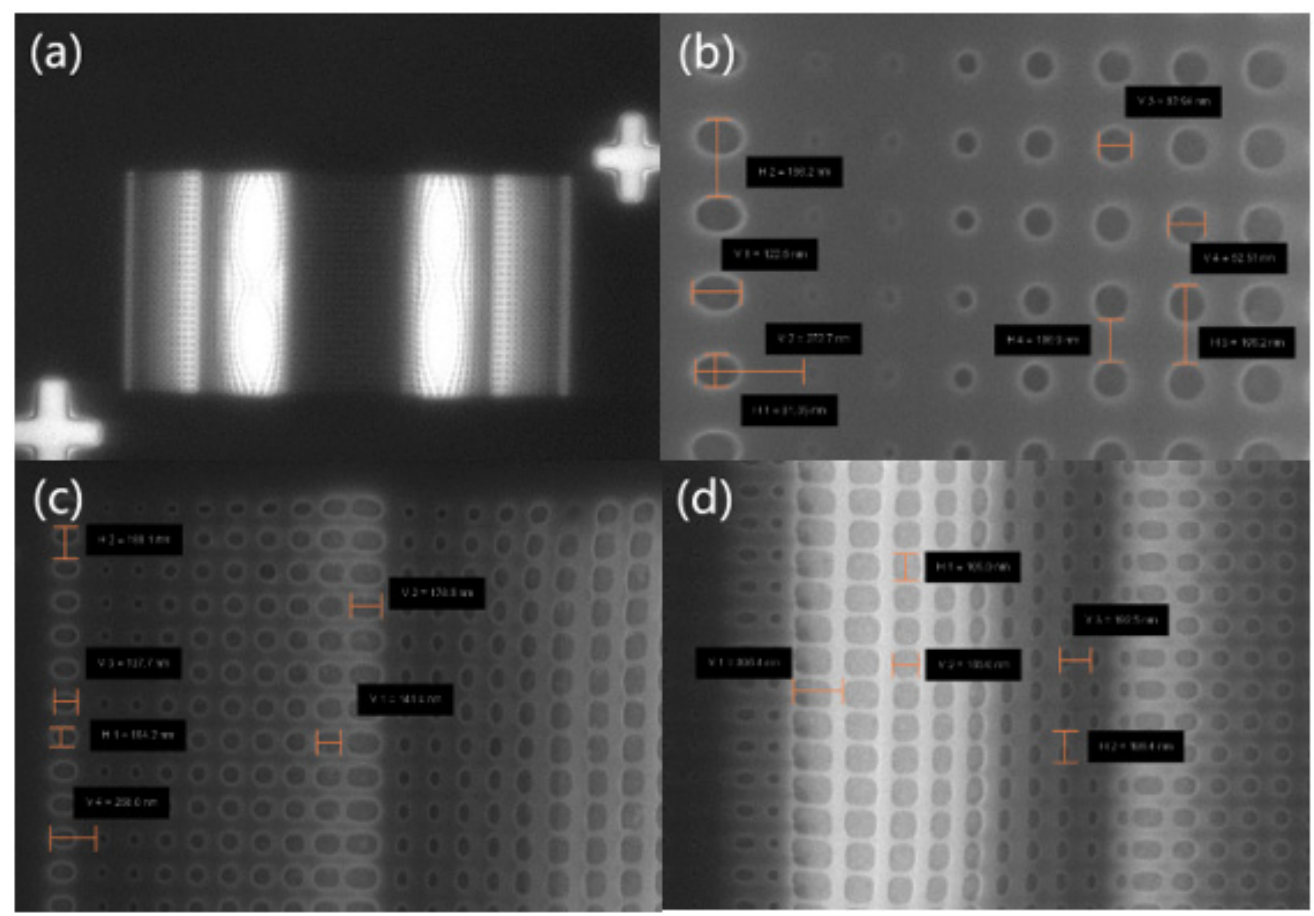

Figure 4. SEM images of different exposure doses: (a) the SEM image with an exposure dose of $700 \mu \mathrm{C} / \mathrm{cm}^{2}$; (b) the SEM image with an exposure dose of $400 \mu \mathrm{C} / \mathrm{cm}^{2}$; (c) the SEM image with an exposure dose of $500 \mu \mathrm{C} / \mathrm{cm}^{2}$; (d) the SEM image with an exposure dose of $600 \mu \mathrm{C} / \mathrm{cm}^{2}$. 


\section{Results and Discussion}

SEM was used to measure the processed metalens structure, and FDTD software was used to build a model based on the processed metalens, simulating and analyzing its focusing characteristics. X-polarized light, y-polarized light, 45-degree linearly polarized light, and the circularly polarized light incidence with a wavelength of $800 \mathrm{~nm}$ were simulated, and the resulting reflected light fields are shown in Figure 5a,c,e,g, respectively. The intensity of the focusing spots along the $\mathrm{x}$-direction is shown in Figure $5 \mathrm{~b}, \mathrm{~d}, \mathrm{f}, \mathrm{h}$. For $\mathrm{x}$-polarized light, the focal length is $4.97 \mu \mathrm{m}$, and the full width at half maximum (FWHM) value of the focal spot is calculated to be $0.47 \mu \mathrm{m}$. For y-polarized light, the focal length is $13.5 \mu \mathrm{m}$, and the FWHM value of the focal spot is calculated to be $0.84 \mu \mathrm{m}$. For 45 -degree linearly polarized light, there are two focal points, and the focal lengths are $4.97 \mu \mathrm{m}$ and $13.5 \mu \mathrm{m}$, respectively. For circularly polarized light, there are also two focal points, and the focal lengths are $4.97 \mu \mathrm{m}$ and $13.5 \mu \mathrm{m}$. It was also noted that the focal point intensity of circularly polarized light is twice that of 45-degree linearly polarized light. The results show that the processed metalens structure can focus at different positions when different polarized lights are incident.
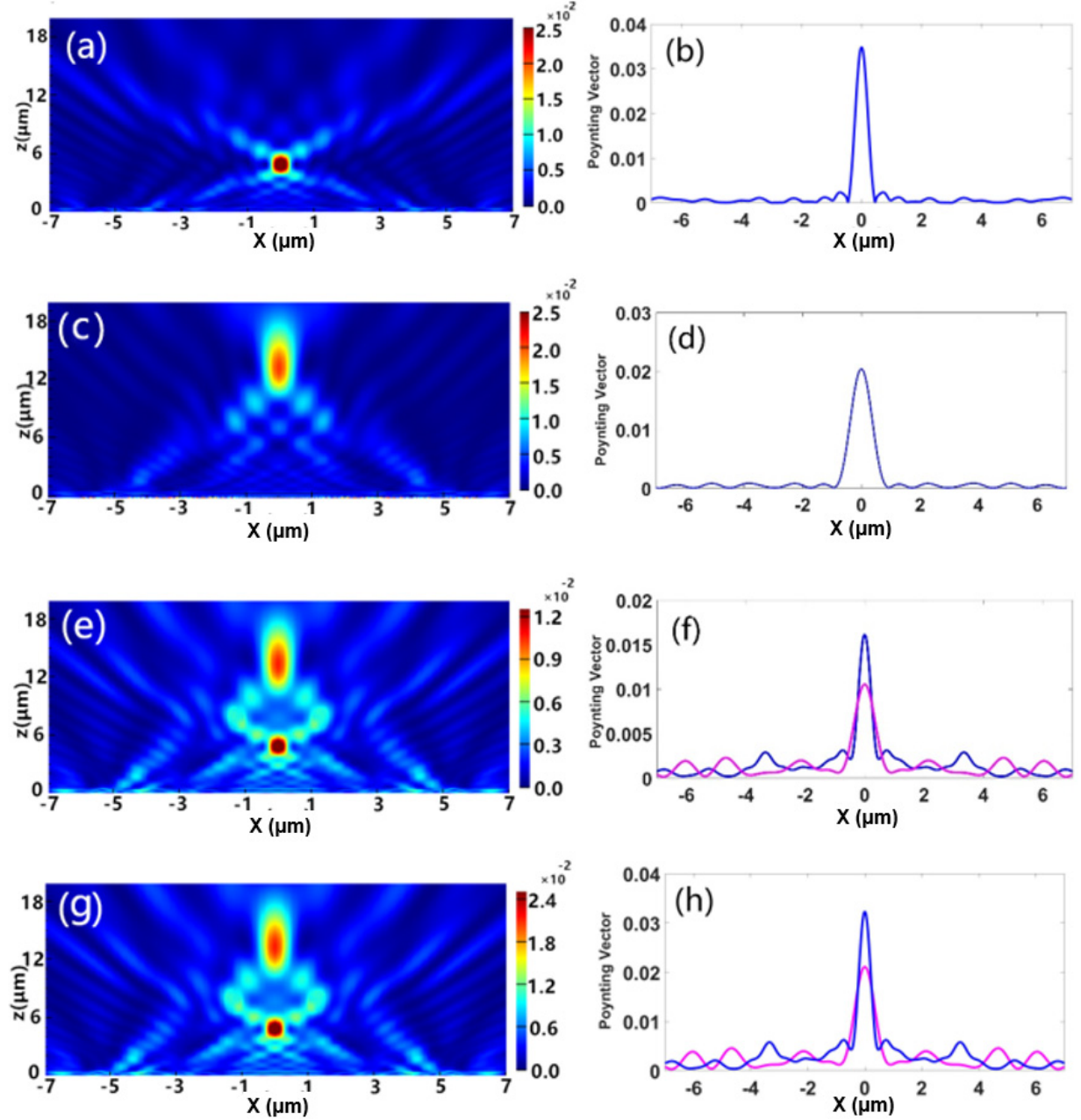

Figure 5. (a) The Poynting vector of the reflected light fields for the metalens with $x$ - polarized incidence; (b) the intensities of the focusing spots along the $\mathrm{x}$-direction for $\mathrm{x}$-polarized incidence; (c) the Poynting vector of the reflected light fields for the metalens with y-polarized incidence; (d) the intensities of the focusing spots along the x-direction for y-polarized incidences; (e) the Poynting vector of the reflected light fields for the metalens with 45-degree linearly polarized incidence; (f) the intensities of the focusing spots along the x-direction for 45-degree linearly polarized incidence; (g) the Poynting vector of the reflected light fields for the metalens with circularly polarized incidence; (h) the intensities of the focusing spots along the x-direction for circularly polarized incidence. 
Then, x-polarized incidences with different wavelengths $(700 \mathrm{~nm}, 750 \mathrm{~nm}, 850 \mathrm{~nm}$, $900 \mathrm{~nm}$ ) were simulated, and the resulting reflected light fields are shown in Figure 6a-d. The results show that the processed metalens structure can also focus light incidences with different wavelengths.
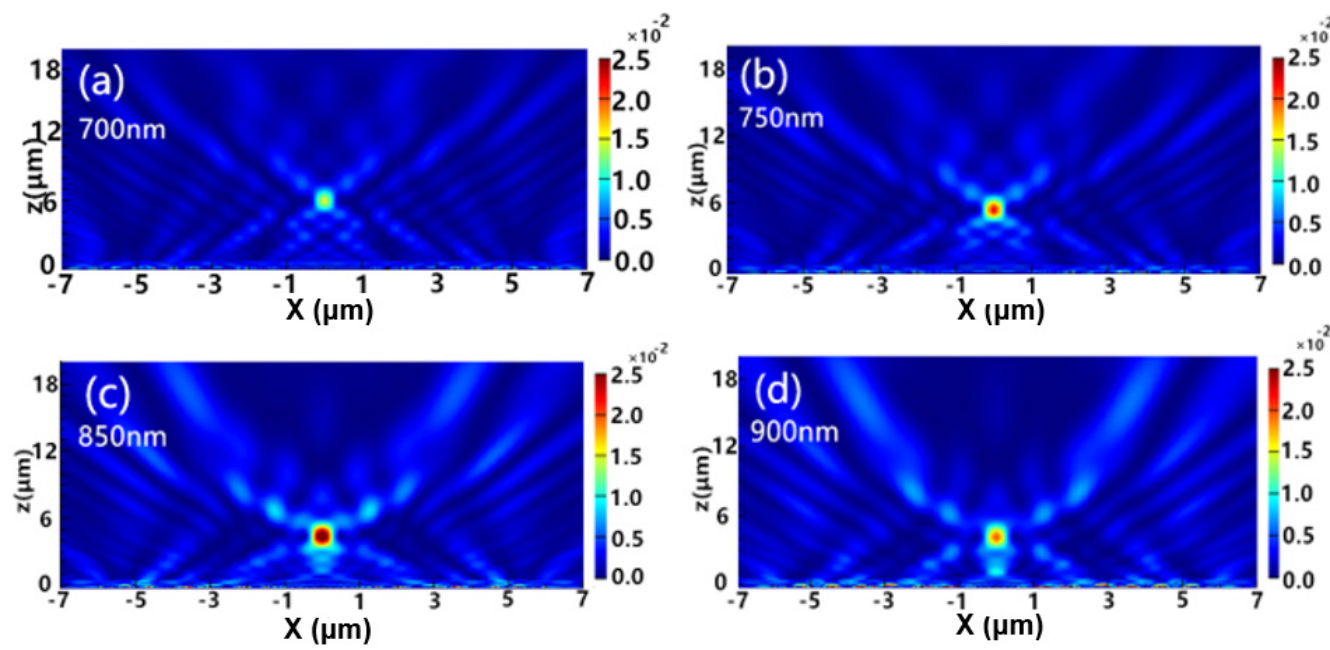

Figure 6. The Poynting vector of the reflected light fields for the metalens with incidence wavelength of (a) $700 \mathrm{~nm},(\mathbf{b}) 750 \mathrm{~nm}$, (c) $850 \mathrm{~nm}$, and (d) $900 \mathrm{~nm}$.

Through modeling and testing of the experimentally prepared metalens structure samples, the results show that the size deviation produced during the processing has little effect on the functionality of the metalens. The processed metalens can also focus different polarized light incidences at different spatial positions and has good focusing effects with different working wavelengths. The processing method of metalens proposed in this paper provides guidance for the preparation of subwavelength metasurface structures.

The limitation of our research is the experimental conditions. We will complete a relevant experiment in the next step.

\section{Conclusions}

In summary, we proposed a metalens structure based on $\mathrm{Au}-\mathrm{MgF}_{2}-\mathrm{Au}$ in infrared waveband and tested it. SEM was used to measure the processed metalens structure, and FDTD software was used to build a model based on the metalens, simulating and analyzing its focusing characteristics. The results, both from simulation and experiment, show that the size deviation produced during the processing has little effect on the functionality of the metalens. The processed metalens can focus different, polarized light incidences at different spatial positions. For x-polarized incidence, the focal length is $4.97 \mu \mathrm{m}$. For $\mathrm{y}^{-}$ polarized incidence, the focal length is $13.5 \mu \mathrm{m}$. For 45 -degree linearly polarized incidence, there are two focal points, and the focal length are $4.97 \mu \mathrm{m}$ and $13.5 \mu \mathrm{m}$, respectively. For circularly polarized incidence, there are also two focal points, and the focal point intensity is twice that of 45-degree linearly polarized light. The processed metalens also has good focusing effects with different working wavelengths. Compared with previous studies, the processed metalens in this study is more functional. We believe that the processing method of metalens proposed in this paper provides guidance for the preparation of subwavelength metasurface structures, and our findings are beneficial in developing new methods of nearinfrared regime manipulation. Experimental characterization and detailed discussion on comparison with the calculation are essential. We will complete a relevant experiment in the next step. 


\begin{abstract}
Author Contributions: Conceptualization, Y.Z. (Yuhui Zhang) and Y.F.; methodology, Y.Z. (Yuhui Zhang); software, Y.Z. (Yuhui Zhang); validation, Y.Z. (Yuhui Zhang), Y.F. and C.M.; formal analysis, Y.Z. (Yuhui Zhang) and B.Y.; investigation, Y.Z. (Yuhui Zhang); resources, Y.Z. (Yuhui Zhang); data curation, Y.Z. (Yuhui Zhang); writing—original draft preparation, Y.Z. (Yuhui Zhang); writingreview and editing, Y.Z. (Yuhui Zhang), Y.F., C.M., B.Y. and Y.Z. (Yuanzhi Zhao); visualization, Y.Z. (Yuhui Zhang); supervision, Y.Z. (Yuhui Zhang); project administration, Y.Z. (Yuhui Zhang); funding acquisition, Y.F. and C.M. All authors have read and agreed to the published version of the manuscript.
\end{abstract}

Funding: This study received funding from "111" Project of China (D17017), Jilin Province Science and Technology Development Plan Project (20200403107SF), Jilin Province Science and Technology Development Plan Project (20210204191YY), and Changchun University of Science and Technology Youth Science Fund Project (XQNJJ-2017-08).

Institutional Review Board Statement: Not Applicable.

Informed Consent Statement: Not Applicable.

Data Availability Statement: Not applicable.

Conflicts of Interest: The authors declare no conflict of interest.

\title{
References
}

1. Smith, D.R.; Pendry, J.B.; Wiltshire, M.C.K. Metamaterials and Negative Refractive Index. Science 2004, 305, 788-792. [CrossRef]

2. Chen, H.T.; Taylor, A.J.; Yu, N.F. A review of metasurfaces: Physics and applications. Rep. Prog. Phys. 2016, 79, 076401. [CrossRef]

3. Lin, D.M.; Fan, P.Y.; Hasman, E.; Brongersma, M.L. Dielectric gradient metasurface optical elements. Science 2014, 345, $298-302$. [CrossRef]

4. Ding, J.; Arigong, B.; Ren, H. Efficient multiband and broadband cross polarization converters based on slotted L-shaped nanoantennas. Opt. Express 2014, 22, 29143-29151. [CrossRef]

5. $\quad \mathrm{Xu}, \mathrm{J}$; Li, R.Q.; Wang, S.Y.; Han, T.S. Ultra-broadband linear polarization converter based on anisotropic metasurface. Opt. Express 2018, 26, 26235-26241. [CrossRef]

6. Jing, X.F.; Cui, G.X.; Zhou, P.W.; Hong, Z. Physical Explanation of Fabry-Perot Cavity for Broadband Bilayer Metamaterials Polarization Converter. J. Lightwave Technol. 2018, 36, 2322-2327. [CrossRef]

7. Peng, Y.; Zang, X.F.; Zhu, Y.M.; Shi, C. Ultra-broadband terahertz perfect absorber by exciting multi-order diffractions in a double-layered grating structure. Opt. Express 2015, 23, 2032-2039. [CrossRef]

8. Yao, G.; Ling, F.; Yue, J.; Luo, C.Y. Dual-band tunable perfect metamaterial absorber in the THz range. Opt. Express 2016, 24, 1518-1527. [CrossRef]

9. Ning, Y.Y.; Dong, Z.W.; Si, J.N.; Deng, X.X. Tunable polarization-independent coherent perfect absorber based on a metal-graphene nanostructure. Opt. Express 2017, 25, 32467-32474. [CrossRef]

10. Kildishev, A.V.; Boltasseva, A.; Shalaev, V.M. Planar photonics with Metasurfaces. Science 2013, 339, 1232009. [CrossRef]

11. Yu, N.F.; Capasso, F. Flat optics with designer metasurfaces. Nat. Mater. 2014, 13, 139-150. [CrossRef] [PubMed]

12. Zhang, Y.H.; Zhao, J.X.; Zhou, J.H.; Liu, Z.Y.; Fu, Y.G. Switchable polarization selective terahertz wavefront manipulation in a graphene metasurface. IEEE Photonics J. 2019, 11, 1. [CrossRef]

13. Zhang, Y.H.; Ma, L.F.; Liu, Z.Y.; Fu, Y.G. Graphene ribbon based tunable terahertz metalens for dual polarization incidences. Opt. Mater. 2019, 97, 109325. [CrossRef]

14. Zhang, Y.H.; Yang, B.W.; Liu, Z.Y.; Fu, Y.G. Polarization Controlled Dual Functional Reflective Planar Metalens in Near Infrared Regime. Coatings 2020, 10, 389. [CrossRef]

15. Li, K.; Guo, Y.H.; Pu, M.B.; Li, P. The dispersion controlling metalens at visible frequency. Opt. Express 2017, 25, 21419. [CrossRef]

16. Ma, Y.B.; Rui, G.H.; Gu, B.; Cui, Y.P. Trapping and manipulation of nanoparticles using multifocal optical vortex metalens. Sci. Rep. 2017, 7, 14611. [CrossRef]

17. Wang, W.; Guo, Z.Y.; Zhou, K.; Sun, Y.X.; Shen, F. Polarization-independent longitudinal multi-focusing metalens. Opt. Express 2015, 23, 29855-29866. [CrossRef]

18. Tian, S.N.; Guo, H.M.; Hu, J.B.; Zhuang, S.L. Dielectric longitudinal bifocal metalens with adjustable intensity and high focusing efficiency. Opt. Express 2019, 27, 680-688. [CrossRef]

19. Lee, B.; Kim, C.; Kim, S.J. Doublet metalens design for high numerical aperture and simultaneous correction of chromatic and monochromatic aberrations. Opt. Express 2020, 28, 18059-18076.

20. Yu, Y.; Li, M.; Chen, R.; Tsai, D.P.; Chin, L.K. Dual-layer Achromatic Metalens Design with an Effective Abbe Number. Opt. Express 2020, 28, 26041-26055.

21. Ammarullah, M.I.; Afif, I.Y.; Maula, M.I.; Winarni, T.I.; Tauviqirrahman, M.; Akbar, I.; Basri, H.; Heide, E.; Jamari, J. Tresca Stress Simulation of Metal-on-Metal Total Hip Arthroplasty during Normal Walking Activity. Materials 2021, 14, 7554. [CrossRef] [PubMed] 
22. Jamari, J.; Ammarullah, M.I.; Saad, A.P.; Uddin, M.; Heide, E.; Basri, H. The Effect of Bottom Profile Dimples on the Femoral Head on Wear in Metal-on-Metal Total Hip Arthroplasty. J. Funct. Biomater. 2021, 12, 38. [CrossRef] [PubMed]

23. Johnson, P.B.; Christy, R.W. Optical Constants of the Noble Metals. Phys. Rev. B (Solid State) 1972, 6, 4370-4379. [CrossRef]

24. Sun, S.; Yang, K.Y.; Wang, C.M.; Juan, T.K.; Tsai, D.P. High-Efficiency Broadband Anomalous Reflection by Gradient Meta-Surfaces. Nano Lett. 2012, 12, 6223-6229. [CrossRef] 